\title{
Two-body Dirac equation with a scalar linear potential
}

\author{
P. Leal Ferreira \\ Instituto de Física Teórica, Universidade Estadual Paulista, Rua Pamplona 145, 01405 São Paulo, São Paulo, Brazil
}

(Received 14 March 1988)

\begin{abstract}
A semirelativistic equation which describes the relative motion of a Dirac particle and its antiparticle, interacting through a scalar potential, linearly dependent on the relative distance, is investigated. The simplest case, corresponding to ${ }^{1} S_{0}$ and ${ }^{3} P_{0}$ solutions, of total angular momentum $J=0$ and positive and negative parities, respectively, is easily worked out for massless constituents. The case of massive constituents, which gives rise to a different regime, is also examined together with other salient features of our approach. A treatment of the light- and heavy-meson $J=0$ sectors is briefly discussed.
\end{abstract}

Phenomenological potential models based on the Dirac equation with a scalar central potential, linearly rising with distance, have been proposed in the literature. ${ }^{1}$ Such potentials give rise to confinement since the Dirac particle gets heavier and heavier when its separation from the fixed center increases indefinitely. Furthermore, the confinement by a scalar potential is also known to be relativistically consistent. By this we mean that it is free of the disease, consisting in the tunneling of the particle through the otherwise confining potential, known as the Klein paradox. ${ }^{2}$

We note, on the other hand, that relativistic potential models have been applied to investigate the hadronic structure under the assumption that the constituent quarks (and/or antiquarks) are spin- $\frac{1}{2}$ Dirac particles which, in a first approximation, move independently of each other inside the confining potential. An obvious feature of this "independent quark approach" is the inherent necessity of subtracting the spurious energy due to the center-of-mass motion of the system. ${ }^{3}$ In the present work the need of these center-of-mass corrections is circumvented $a b$ initio if we restrict ourselves to mesons, described by a two-body Dirac equation formulated in the center-of-mass frame of the quark-antiquark system. As we shall see, such an approach, when implemented by the introduction of Lorentz-scalar linear potential terms for both a quark and an antiquark, seems to provide a convenient basis for a treatment of mesons in which relativistic effects are incorporated in a simple and direct way.

Having this in mind, let us consider a system consisting of a spin- $\frac{1}{2}$ Dirac particle (say 1) and its antiparticle (say 2 ), of common rest mass $m$ and obeying, in the center-ofmass reference system, the equation $(\hbar=c=1)$

$$
\begin{aligned}
{\left[-i \alpha_{1} \cdot \nabla+\beta_{1}(m+\lambda r)\right.} & +i \alpha_{2} \cdot \nabla \\
& \left.+\beta_{2}(m+\lambda r)-W\right] \psi(\mathbf{r})=0 .
\end{aligned}
$$

In Eq. (1), a linear scalar potential $\lambda r$ is introduced in both particle and antiparticle mass terms. The operator $-i \nabla$ appearing in it is the momentum conjugate to $\mathbf{r}=\mathbf{r}_{1}-\mathbf{r}_{2}$ and $r=|\mathbf{r}|$. The wave function $\psi(\mathbf{r})$ is a 16component spinor $\psi_{i j}$, the subscripts $i$ and $j$ $(i, j=1,2,3,4)$ referring to particle (1) and antiparticle (2), respectively. We note that Eq. (1) may be considered as a special case of the Kemmer equation ${ }^{4}$ which was also applied by Fermi and $\mathrm{Yang}^{5}$ to pions, regarded as a bound state of a nucleon and a antinucleon.

We shall confine our attention here to solutions of Eq. (1) corresponding to $J=0, J$ being the total angular momentum of the system. These solutions are labeled as ${ }^{1} S_{0}$ and ${ }^{3} P_{0}$ and have negative and positive parities, respectively.

Several formalisms ${ }^{6}$ exist in order to derive the radial equations relative to $\mathrm{Eq}$. (1). For the present purposes it is reasonable to adopt the more compact treatment given by Moseley and Rosen ${ }^{7}$ and Nagasaki. ${ }^{8}$ From their work, we know that there are five linear combinations involving the 16 components $\psi_{i j}$, which are nonvanishing for the ${ }^{1} S_{0}$ state. With respect to rotations, they are two scalars denoted by $I$ and $A_{4}$, and one vector $F=\left(F_{1}, F_{2}, F_{3}\right)$. They obey the set of equations

$$
\begin{aligned}
& W I+2 i \nabla \cdot \mathbf{F}+4(m+\lambda r) A_{4}=0, \\
& W \mathbf{F}+2 i \nabla I=0, \quad W A_{4}+4(m+\lambda r) I=0,
\end{aligned}
$$

where

$$
I=f_{1}(r), \quad A_{4}=f_{2}(r), \quad \mathbf{F}=f_{3}(r) \frac{\mathbf{r}}{r} .
$$

It is easily seen that (2) yields the following system of radial equations:

$$
\begin{aligned}
& W f_{1}(r)+2 i\left(r \frac{d}{d r}+3\right) \frac{1}{r} f_{3}(r)+2(m+\lambda r) f_{2}(r)=0, \\
& W f_{2}(r)+2(m+\lambda r) f_{1}(r)=0, \\
& W f_{3}(r)+2 i \frac{d}{d r} f_{1}(r)=0 .
\end{aligned}
$$

By eliminating the components $f_{2}$ and $f_{3}$, one gets (with a prime denoting $d / d r$ ) 


$$
u^{\prime \prime}+\frac{W^{2}}{4}\left[1-4 \frac{(m+\lambda r)^{2}}{W^{2}}\right] u(r)=0,
$$

where we set $u(r)=r f_{1}(r)$.

The energy eigenvalues $W$ are obtained by means of the boundary conditions

$$
u(0)=0, u(\infty)=0 .
$$

For simplicity, we shall restrict our discussion to massless constituents $(m=0)$. In this case it is interesting to point out that, although we started from linear potentials in Eq. (1), the radial Eq. (5) corresponds to the radial Schrödinger equation for a three-dimensional isotropic harmonic oscillator, in the $S$ wave. This feature is clearly a consequence of the relativistic kinematics in which Eq. (1) was formulated.

The eigenfunctions $u(r)$, satisfying conditions (6), are given in standard form as

$$
u(r)=r \exp \left(-\frac{\lambda}{2} r^{2}\right){ }_{1} F_{1}\left(\frac{1}{2}\left(\frac{3}{2}+a\right), \frac{3}{2} ; \lambda r^{2}\right) .
$$

In Eq. (7),

$$
-a=\frac{W^{2}}{8 \lambda}=2 n_{r}+\frac{3}{2}
$$

where $n_{r}$ is a non-negative integer $\left(n_{r}=0,1,2, \ldots\right)$. The corresponding eigenvalues $W$ are given by

$$
\frac{W}{\sqrt{\lambda}}=2\left(4 n_{r}+3\right)^{1 / 2} \text {. }
$$

The ${ }^{1} S_{0}$ states, Eq. (3), are normalized by means of the relation

$$
4 \pi \mathcal{N}^{2} \int_{0}^{\infty}\left(\left|f_{1}\right|^{2}+\left|f_{2}\right|^{2}+\left.f_{3}\right|^{2}\right) r^{2} d r=1
$$

For the lowest-energy state $\left(n_{r}=0\right)$, one finds $\mathcal{N}=\left(\lambda^{3} / 4 \pi^{3}\right)^{1 / 4}$. We note from (9) that $W$ goes to zero as the interaction is cut out adiabatically to zero, as expected.

The state ${ }^{3} P_{0}$, of positive parity, can be worked out in a similar way, ${ }^{6,7}$ in terms of seven linear combinations, denoted as $J=f_{4}(r), \mathbf{G}=f_{s}(r) \mathbf{r} / r$, and $\mathbf{U}=f_{6}(r) \mathbf{r} / r$. We get, with $g(r)=r f_{5}(r)$, the radial equation

$g^{\prime \prime}-\frac{2}{r^{2}} g(r)+\frac{W^{2}}{4}\left[1-4 \frac{(m+\lambda r)^{2}}{W^{2}}\right] g(r)=0$,

with the conditions $g(0)=g(\infty)=0$.

We note that Eq. (10) differs from Eq. (5) only by the presence of a $P$-wave centrifugal term. Again, for massless constituents, the radial wave function $g(r)$ is of the form

$$
g(r)=r^{2} \exp \left[-\frac{\lambda}{2} r^{2}\right){ }_{1} F_{1}\left(\frac{1}{2}\left(\frac{5}{2}+a\right), \frac{5}{2} ; \lambda r^{2}\right),
$$

corresponding to a tower of $P$-wave energy states

$$
\frac{W}{\sqrt{\lambda}}=2\left(4 n_{r}+5\right)^{1 / 2} \text {. }
$$

Our next step is to consider massive constituents. It is easy to see that the solutions of Eq. (5) can then be expressed in terms of parabolic cylinder functions ${ }^{9} D_{v}(z)$ :

$$
u(r)=D_{v}(z),
$$

with $z=(2 \lambda)^{1 / 2}(r+m / \lambda)$ and $v=W^{2} / 8 \lambda-\frac{1}{2}$.

Thus, in view of (7), the energy eigenvalues can be obtained, for $m>0$, as solutions of the equation

$$
D_{v}(m \sqrt{2 / \lambda})=0 \text {. }
$$

It is remarkable that the massive regime departs from the harmonic behavior, characteristic of the massless case, being described by the positive zeros of the parabolic cylinder functions, Eq. (14). In other words, as far as $J=0$ solutions are concerned, we predict that light mesons and the different families of heavy mesons correspond to different regimes, associated, respectively, to the massless case and to each one of the massive cases, as discussed above. Similar behavior is also expected for the $J \neq 0$ solutions of Eq. (1) which, however, would require a separate discussion.

We now wish to point out that the present scheme can be extended in a straightforward way by introducing in Eq. (1), as in the case of the Kemmer equation, additional two-body interaction terms ${ }^{4} I_{12}$, each one being characterized by its specific Lorentz nature: $I_{12}=\sum_{k=0}^{5} b_{k} O_{k} V_{k}(r)$. Here, $b_{k}$ are constants, $V_{k}$ are given functions of $r$, and $O_{k}$ are the operators

$$
\begin{aligned}
& O_{1}=\beta_{1} \beta_{2}, \quad O_{2}=\frac{1}{2}\left(1-\alpha_{1} \cdot \alpha_{2}\right) \\
& O_{3}=\frac{1}{2} \beta_{1} \beta_{2}\left(\sigma_{1} \cdot \sigma_{2}+\alpha_{1} \cdot \alpha_{2}\right) \\
& O_{4}=\frac{1}{2}\left(\sigma_{1} \cdot \sigma_{2}-\Gamma_{1} \Gamma_{2}\right)
\end{aligned}
$$

and

$$
O_{5}=\beta_{1} \beta_{2} \Gamma_{1} \Gamma_{2}
$$

with $\boldsymbol{\sigma}=(i / 2) \boldsymbol{\alpha} \times \boldsymbol{\alpha}$, and $\Gamma=-i \alpha_{x} \alpha_{y} \alpha_{z}$.

In brief, our prescription here is to treat the Kemmer interaction terms at the level of first-order perturbation theory, in order to avoid spurious singularities in the radial equations which would destroy the normalizability of the solutions, as discussed by Childers ${ }^{10}$ in a similar context, involving Breit interaction terms.

Finally, we remark that a generalization of Eq. (1) for the case of constituents of unequal masses can also be performed similarly. ${ }^{8}$ This corresponds to having a system consisting of a Dirac particle of given type and mass $m_{1}$ and an antiparticle of another type, of mass $m_{2}$, a typical instance being, of course, the kaons belonging to the $0^{-}$pseudoscalar light-meson sector. For the particular instance of ${ }^{1} S_{0}$ solutions, instead of the radial Eq. (5), we now get

$$
\begin{aligned}
u^{\prime \prime}+\frac{1}{4}[W- & \left.\frac{\left(m_{1}-m_{2}\right)^{2}}{W}\right] \\
& \times\left(W-\frac{1}{W}\left(m_{1}+m_{2}+2 \lambda r\right)^{2}\right) u=0 .
\end{aligned}
$$

In the so-called one-body limit, when $W=W_{1}+m_{2}$ 
and $m_{1} / m_{2}<1$, Eq. (15) results in $u^{\prime \prime}+W_{1}\left(W_{1}\right.$ $-2 \lambda r)=0$, an equation which can be readily solved in terms of the Airy function $\operatorname{Ai}(z)$. One finds for $W_{1}$ the expression

$$
\frac{W_{1}}{\sqrt{\lambda}}=\sqrt{2}\left|a_{n}\right|^{3 / 4},
$$

where $a_{n}$ is the $n$th zero of the function $\operatorname{Ai}(z)$, $n=1,2, \ldots$.

In conclusion, the following remarks are in order. If it is assumed that the $\eta(550) 0^{-}$state of the pseudoscalar light-meson sector is described by the ${ }^{1} S_{0}$ solution Eq. (3), then its correct mass can be obtained by taking $\sqrt{\lambda}=0.158 \mathrm{GeV}$, in the massless regime of ordinary $u$ and $d$ quarks. With the same value of $\lambda$, the kaon, as described by a ${ }^{1} S_{0}$ negative-parity solution, Eq. (15), acquires its correct mass for $m_{1}=0$ and a strange-quark mass $m_{2}=0.461 \mathrm{GeV}$. Notice that, by using Eq. (14), we can get the mass of the $\eta_{c} 0^{-}$state of the $J / \psi$ family again with the same $\lambda$ and a charmed-quark mass of 1.35 $\mathrm{GeV}$.

Finally, we remark that an adequate choice of the two-body relativistic interaction terms $I_{12}$ is required to obtain the spin-dependent interactions responsible for a correct description of the fine splitting of the ${ }^{3} P_{J}$ states in quarkonia and, particularly, of the transition rates for radiative decays involving heavy-meson excitations in both $J / \psi$ and $\Upsilon$ spectroscopies. ${ }^{11}$ However, a discussion of such topics, in the light of the present approach, requires a detailed discussion of the $J \neq 0$ solutions of Eq. (1), which are outside of the limited scope of this work.

Conversations with Dr. L. Tomio and B. E. Palladino are gratefully acknowledged.
${ }^{1}$ C. L. Critchfield, Phys. Rev. D 12, 923 (1975); J. Math. Phys. 17, 281 (1976); D. W. Rein, Nuovo Cimento 38, 19 (1977).

${ }^{2}$ P. M. Fishbane, S. G. Gasiorowicz, D. C. Johannsen, and P. Kaus, Phys. Rev. D 27, 2433 (1983).

3J. F. Donoghue and K. Johnson, Phys. Rev. D 21, 1975 (1980); R. Tegen, R. Brockmann, and W. Weise, Z. Phys. A 307, 339 (1982); B. E. Palladino and P. Leal Ferreira, Phys. Rev. D 34, 2168 (1986).

${ }^{4}$ N. Kemmer, Helv. Phys. Acta 10, 48 (1937).

${ }^{5}$ E. Fermi and C. N. Yang, Phys. Rev. 76, 1739 (1949).

${ }^{6}$ Y. Koide, Prog. Theor. Phys. 37, 1337 (1967); 39, 817 (1968); W. Krolikowski and J. Rzewuski, Acta Phys. Pol. B 7, 487 (1976); H. Joos, J. Leal Ferreira, and A. H. Zimerman, Nuovo
Cimento 5, 57 (1957); J. Leal Ferreira and A. H. Zimerman, An. Acad. Bras. Cienc. 30, 281 (1958).

${ }^{7}$ H. M. Moseley and N. Rosen, Phys. Rev. 80, 177 (1950).

${ }^{8}$ M. Nagasaki, Prog. Theor. Phys. 37, 437 (1967).

${ }^{9}$ W. Magnus and F. Oberhettinger, Functions of Mathematical Physics (Chelsea, New York, 1954), p. 91.

${ }^{10}$ R. W. Childers, Phys. Rev. D 26, 2902 (1982).

${ }^{11}$ See, for instance, J. Lee-Franzini, in Physics of Quarkonia, proceedings of the International Symposium on Lepton and Photon Interactions at High Energies, Hamburg, West Germany, 1987, edited by R. Rueckl and W. Bartel [Nucl. Phys. B, Proc. Suppl. 3, 139 (1987)]. 